\title{
Pancreatic Mucinous Cystic Neoplasm Communicating with Main Pancreatic Duct: An Unrecognized Presentation of Pancreatic Mucinous Neoplasm?
}

\author{
WEIXUN ZHOU ${ }^{1,2}$, TRUSTIN SAAM $^{3}$, YIHUA ZHOU ${ }^{3}$, JOSE TREVINO $^{4}$, \\ XIULI LIU ${ }^{2}$, DENGFENG CAO ${ }^{5}$ and JINPING LAI ${ }^{2}$ \\ ${ }^{1}$ Department of Pathology, Peking Union Medical College Hospital, \\ Chinese Academy of Medical Sciences, Beijing, P.R. China; \\ ${ }^{2}$ Department of Pathology, Immunology, and Laboratory Medicine, \\ University of Florida College of Medicine, Gainesville, FL, U.S.A.; \\ ${ }^{3}$ Department of Radiology, Saint Louis University School of Medicine, St. Louis, MO, U.S.A.; \\ ${ }^{4}$ Department of Surgery, University of Florida College of Medicine, Gainesville, FL, U.S.A.; \\ ${ }^{5}$ Department of Pathology, Immunology, and Laboratory Medicine, \\ Washington University in Saint Louis, St. Louis, MO, U.S.A.
}

\begin{abstract}
Mucinous cystic neoplasms (MCNs) and intraductal papillary mucinous neoplasms (IPMNs) are two well recognized entities of precursor cystic lesions of pancreatic duct adenocarcinoma. The characteristic features of MCNs are the lined mucinous epithelium with underlying ovarian-type stroma, but without communication with the ducts, while that for IPMNs are the communication with the ducts but without the underlying ovarian-type stroma. Here we report a case of MCN communicating with the main pancreatic duct in a 68-year-old woman. The initial radiographic diagnosis was pancreatic IPMN with main pancreatic involvement and this was also confirmed during gross examination. Histologically, the pancreatic cystic neoplasm was lined with mucinous epithelium with underlying ovarian-type of stroma. Immunohistochemical stains confirmed that the stroma cells were positive for $E R$, $P R$, alpha-inhibin and focally positive for CD10. The final pathologic diagnosis was pancreatic mucinous cystic neoplasm communicating with the main pancreatic duct. To the best of our knowledge, this is the second pathology confirmed case of MCN communicating with the main pancreatic duct. A careful gross examination and bivalvation
\end{abstract}

Correspondence to: Prof. Jinping Lai, Department of Pathology, Immunology, and Laboratory Medicine, University of Florida College of Medicine, Gainesville, FL 32610, U.S.A. Tel: +1 3526279240, Fax: +1 3526279242, e-mail: jinpinglai@ufl.edu

Key Words: Pancreatic mucinous cystic neoplasm, MCN, main pancreatic duct. of the main duct communicating with the cystic neoplasm helps render the correct diagnosis. If more cases are reported in the future, the MCN communicating with duct could become a new entity of pancreatic mucinous neoplasm.

Pancreatic cystic neoplasms are a heterogeneous group of tumors. Among them, both mucinous cyst neoplasms (MCNs) and intraductal papillary mucinous neoplasms (IPMNs) are well recognized to be precursor lesions of pancreatic duct adenocarcinoma. MCNs and IPMNs have different characteristics. MCNs almost exclusively occur in women, with a mean age of 45 years (1). Benign behaving MCNs are mostly located in pancreatic body and tail, while malignant MCNs are located more often in the head of the pancreas $(2,3)$. MCNs could be multilocular or unilocular, but classically do not communicate with the pancreatic ductal system $(3,4)$. Microscopically, the characteristic feature of MCN is ovarian-type stroma beneath the mucinous epithelium $(1,4)$. In contrast, IPMNs affect men and women equally and are mostly diagnosed between 60 and 70 years of age. IPMNs involve either main duct or side branch ducts or both and show cystic dilation of the involved ducts. Approximately half of IPMNs are located in the head of the pancreas (3). Histologically, IPMNs, unlike MCNs, are lined with mucin-producing epithelium of different types. According to the epithelium, IPMNs are classified as intestinal, gastric, pancreaticoblliary, and oncocytic subtypes.

IPMNs and MCNs do share some common characteristics, including cystic mass on imaging, less invasiveness, and presence of a spectrum of neoplastic changes. An important feature of IPMNs is their communication with pancreatic 

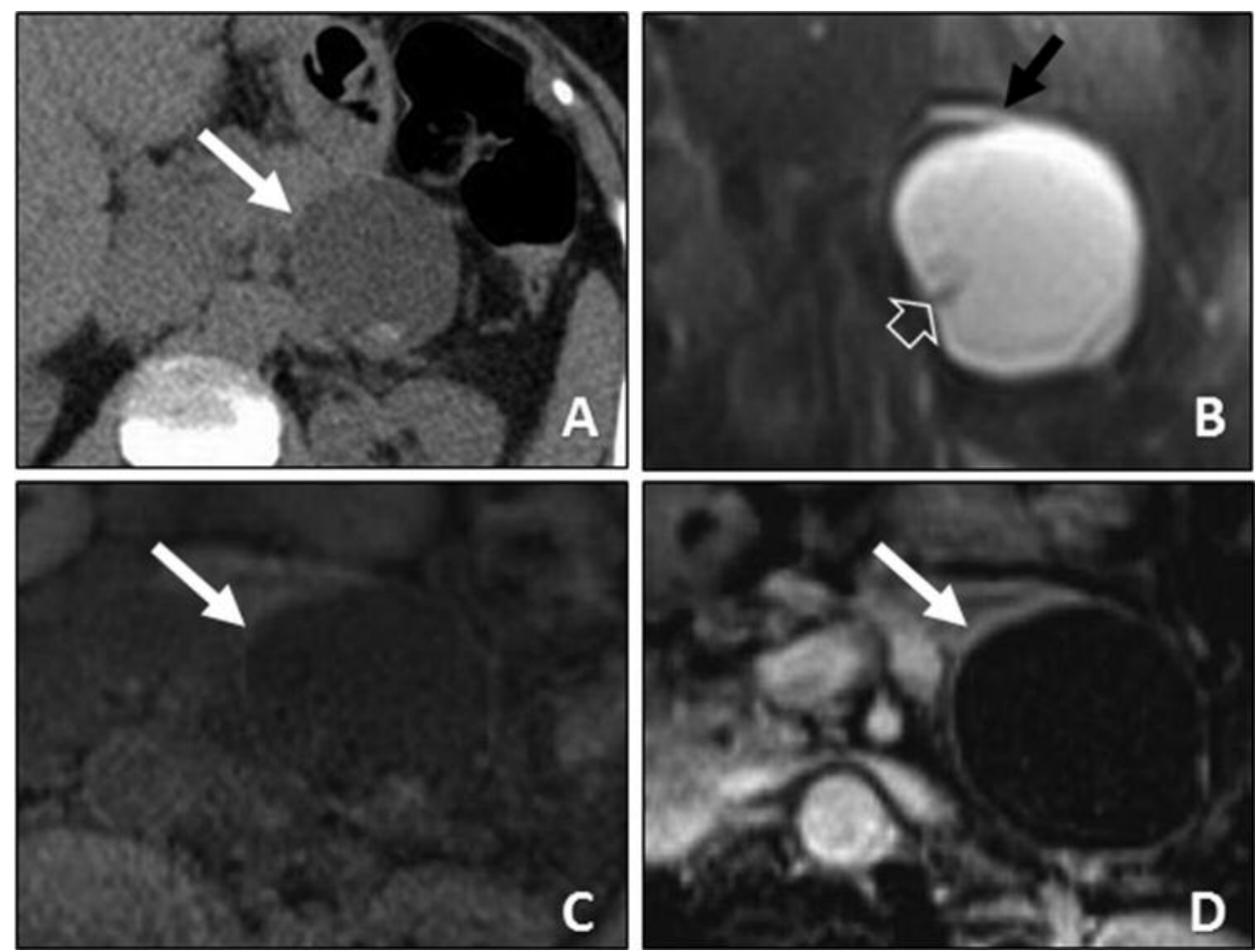

Figure 1. The radiographic changes of the lesion. A: CT demonstrates a $4.6 \times 4.1 \mathrm{~cm}$ cystic structure (white arrow) in the junction of the pancreatic body and the tail. B: MRCP demonstrates a direct communication between the main pancreatic duct (black arrow) and the cyst. There is a small internal septation within the inferior aspect of the cyst (open arrow). C: Pre-contrast T1 weighted image shows the cyst (white arrow) with a thin and smooth wall. D: Post-contrast T1 weighted image demonstrates no abnormal enhancement associated with the cyst (white arrow).

ducts. An important feature of MCNs is their underlying ovarian-type stroma without communication to the pancreatic ducts. These features are pathologically used to distinguish one cystic neoplasm from the other. Cases of MCNs communicating with either main duct or side branch duct are rarely reported and could be under recognized. To the best of our knowledge, only one case has previously been reported to have a pancreatic cyst lesion with features of both IPMN and MCN (5). Here we report the second case of MCN communicating with the main pancreatic duct and discuss the differential diagnosis of the two mucinous neoplasms.

\section{Case Presentation}

A 68-year-old African American female with recently diagnosed chronic kidney disease presented with complaints of non-radiating upper abdominal pain and fever. The patient did not have any other associated symptoms including nausea, vomiting or change in bowel habits. Due to her vague clinical presentation, a computed tomography (CT, Figure 1A) was performed and demonstrated a $4.6 \times 4.1 \mathrm{~cm}$ cystic structure at the junction of the pancreatic body and the tail. An $8 \mathrm{~mm}$ hyperdense focus in the portion of the lesion was noted which likely represented a small focus of calcification. Due to its complexity on the pancreas and possible cystic component with duct involvement, magnetic resonance cholangiopancreatography (MRCP, Figure 1B) was also performed and demonstrated a direct communication between the main pancreatic duct and the cyst. There was a small focus of $\mathrm{T} 2$ hypointensity within the inferior aspect of the cyst which suggested a small internal septation. The pre-contrast T1 weighted image (Figure 1C) showed that the cyst had a thin and smooth wall that was isointense to the pancreas parenchyma, suggesting that the cyst was likely within the pancreatic parenchyma. The postcontrast T1 weighted image (Figure 1D) demonstrated no abnormal enhancement, in and outside of the cyst. The initial radiographic diagnosis was pancreatic IPMN with main pancreatic involvement. An endoscopic ultrasound was performed to further characterize the cystic neoplasm and demonstrated an anechoic lesion suggestive of a cyst in the pancreatic body communicating with the main pancreatic duct, measured $50 \times 50 \mathrm{~mm}$ in maximal cross-sectional 

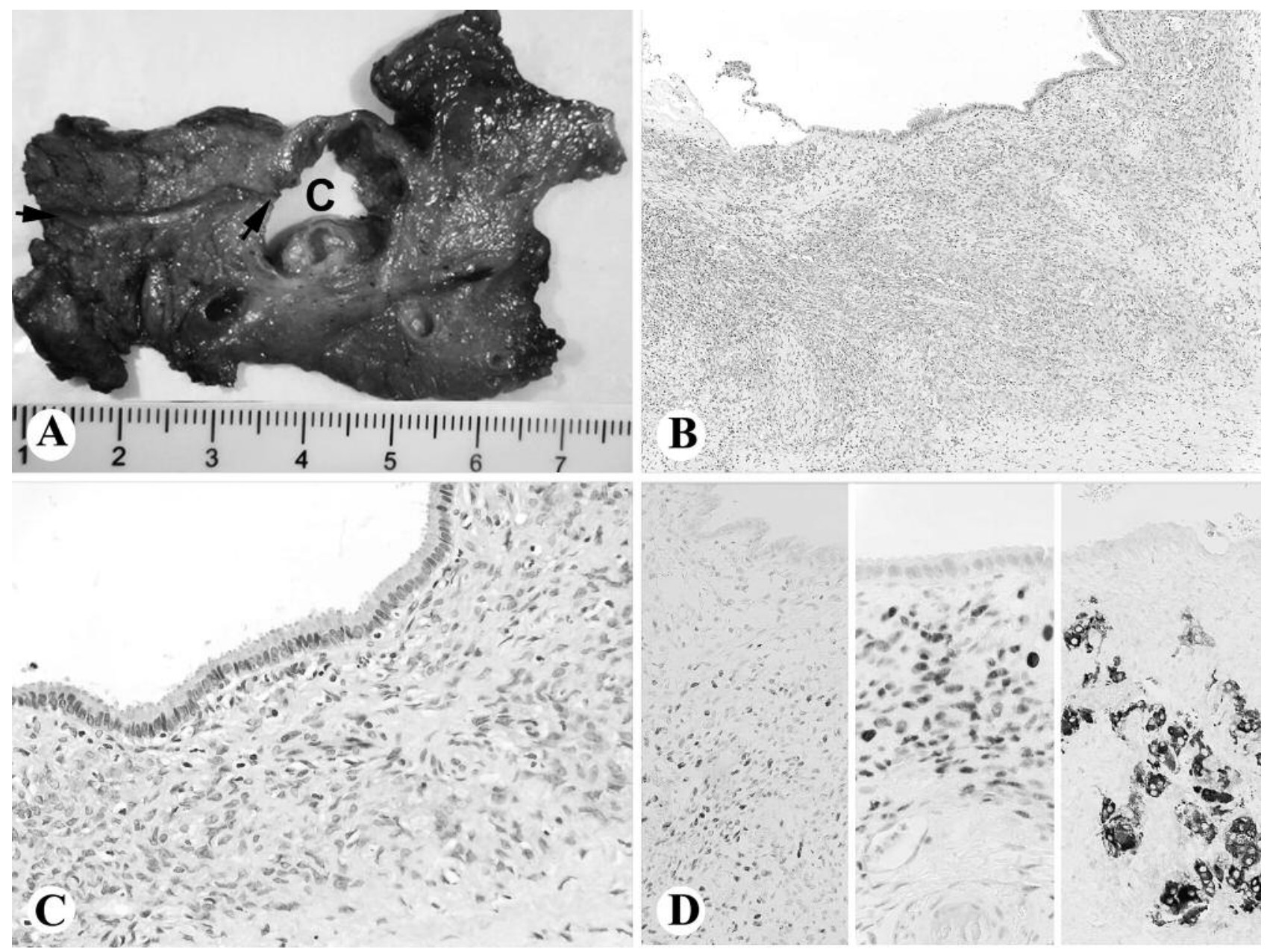

Figure 2. MCN communicating with the main pancreatic duct. A: Grossly, the cystic neoplasm (C) communicating with the main duct (arrows); B$C$ : Histologically, the cyst lined by mucinous epithelium with underlying ovarian-type stroma. (H\&E, B, 100x; C, 400x); D: Immunohistochemistry showing positive ER (left, 200x), PR (middle, 400x), and inhibin (right, 400x) in the stromal cells.

diameter. The pancreatic tail upstream from the cyst had dilated pancreatic duct and atrophic parenchyma. Diagnostic and therapeutic fine needle aspiration for fluid was performed. The fluid was clear and thin. Amylase of the cyst fluid was $65 \mu / 1$, and CEA was $222.7 \mu / 1$.

Due to the features suggesting main-duct IPMN, the patient had a distal pancreatectomy/splenectomy. The resected pancreas measured $12 \mathrm{~cm}$ in length. The main pancreatic duct at the margin had a diameter of $2.0 \mathrm{~mm}$, and it maintained an even diameter of $2.0 \mathrm{~mm}$ for a distance of $3.5 \mathrm{~cm}$ from the proximal margin. The pancreatic duct opened into a smooth-walled cyst (Figure 2A). The cyst measured approximately $3.0 \times 2.5 \times 2.0 \mathrm{~cm}$ when intact and was full of brownish and mucinous contents. This cyst also contained a smooth white stone measuring $0.9 \times 0.5 \times 0.4 \mathrm{~cm}$.
The cyst had two smaller loculations separated by thin and smooth septae. The cyst did not have a distal exit point for the pancreatic duct. Pancreas distal to the cyst had lost its lobulated texture and had fibrosis with yellow speckled areas, compatible with chronic pancreatitis.

Microscopically, the pancreatic cystic neoplasm was lined by mucinous epithelium with underlying ovarian-type cellular stroma (Figures 2B and 2C). No high-grade dysplasia or invasive carcinoma was identified. Immunohistochemical stains showed that the underlying cellular stroma was diffusely positive for ER, PR, alphainhibin (Figure 2D) and focally positive for CD10, consistent with an ovarian-type stroma. No metastasis was identified in the five examined regional lymph nodes. Chronic pancreatitis and a small pseudocyst were seen in the tail of 
the pancreas. The final diagnosis was pancreatic mucinous cystic neoplasm communicating with the main pancreatic duct. One-year follow up, the patient was doing well.

\section{Discussion}

Pancreatic mucinous cystic neoplasms are relatively uncommon. Among individuals without a history of pancreatic disease, the prevalence of pancreatic cysts is about $2.5 \%$ by CT or MRI detection (2). With the development of better imaging technologies, the identification of these premalignant cysts is becoming more prevalent and the incidental findings are present while patients work up for other clinical conditions.

The importance of detection and management of IPMNs and MCNs is that while they can have benign epithelial components, they have the potential for malignant transformation from dysplasia to carcinoma. The risk of malignancy in $\mathrm{MCN}$ is $17 \%-24 \%(2,6)$, and larger $(>3 \mathrm{~cm})$ MCNs have higher risk of malignancy. In IPMNs, rates of malignancy depend on the involvement of ducts. Main duct IPMNs have the highest risk of malignancy, with mean risk around $65 \%$ to $70 \%$. Branch duct IPMNs (BD-IPMNs) have mean risk of malignancy around $25 \%$ (3), The presence of pancreatitis-like symptoms or mural nodule in BD-IPMN is associated with higher and faster malignant risk $(7,8)$.

Management of IPMNs and MCNs is according to the differences in malignancy transformation and growth pattern. Not surprising, surgical resection is recommended for all surgically fit patients with MCN. For IPMNs, it depends on type and size. Resection is recommended for main duct IPMNs (MDIPMN), while BD-IPMN could be followed up. "Worrisome features" of malignancy in IPMNs on imaging are cyst with $\geq 3$ $\mathrm{cm}$, thickened enhanced cystic walls, abrupt change in the main pancreatic duct (MPD) size of 5-9 mm, non-enhanced mural nodules, abrupt change in the main pancreatic duct caliber with distal pancreatic atrophy, and lymphadenopathy. Cysts with symptoms or "worrisome features" should be considered for surgical resection. Patients with cysts of $\leq 3 \mathrm{~cm}$ and without "worrisome features" could be observed without immediate resection. But they have to undergo surveillance of MRI/CT and serologic marker routinely (9).

After resection, surveillance is different for IPMNs and MCNs. Non-invasive MCNs do not require surveillance after complete resection. IPMNs need surveillance based on the resection margin status. If there are no residual lesions, repeat examinations at 2 and 5 years may be reasonable. For patients with low-grade or moderate-grade dysplasia at the margin, history/physical examination and MRCP surveillance at least twice a year is recommended (9). Based on the difference of their malignant transformation risk, treatment and surveillance, it's important to distinguish IPMNs and MCNs on resected specimens.
From their imaging characteristics, MD-IPMN has dilated main duct $\geq 5 \mathrm{~mm}$, which is easier to identify. Both BD-IPMNs and MCNs have focal cysts and are mostly confused (2). Features of BD-IPMN include multiplicity and visualization of a connection to the main pancreatic duct. Though the connection is not always observed, it's a very important differential criterium from MCNs. Analyses of the CEA and amylase levels, and cytology of the cyst may help in detecting malignancy, but cannot distinguish MCN and IPMN (6). In our case, CT and MRCP showed a single cyst with a thin and smooth wall. There was hyperattenuating focus within the dependent portion and small foci of rim calcification along its edges. Marked dilatation of the pancreatic duct was seen within the pancreatic tail. MRCP demonstrates a direct communication between the main pancreatic duct and the cyst. The image findings are suspicious for IPMN. However, gross examination and histologic changes demonstrated a MCN communicating with the main duct.

In many cases, the definite diagnosis relies on histology, like ours. MCNs have mucinous epithelium, which is similar to gastric type epithelium. While IPMNs have mucinous epithelium-lined papillary structure in a cystically dilated ductal system, MCNs could have septa or protrusions similar to papilla, and papillary structure as well. The main difference is the ovarian-type stroma in MCNs (2). By definition, ovariantype stroma is a basic element of MCN in WHO classification (10) and thus is mandatory for diagnosis of MCN (6). In our case, the cystic neoplasm was lined with low-grade mucinous epithelium with underlying cellular stroma. The stroma was confirmed as ovarian-type stroma by its strong immunoreactivity for ER, PR, and alpha-inhibin and focal immunoreactivity for CD10. With this ovarian-type stroma, we diagnosed the case as pancreatic mucinous cystic neoplasm, even though it connected to the main pancreatic duct.

MCNs connecting with the main pancreatic duct is extremely rare. There were some cases of MCNs communicating to MPD in old literature, but that was because of confusion between MCNs and IPMNs (11). After the well recognition of IPMNs, rare cases were reported. Morel et al. reported one case, but only showed the connection by MRI, without confirmation in the resected specimen (11). Masia et al. (5) reported a case of MCN located in main pancreatic duct. It had ovarian-type stroma and located in the dilated main pancreatic duct. Our case is similar to that, $\mathrm{MCN}$ with connection to the main pancreatic duct confirmed by gross examination. However, the main duct connecting with the $\mathrm{MCN}$, in our case, was not dilated. A careful gross examination with bivalvation of the pancreas through a probe connecting the duct and cyst was the key to confirm the physical connect of the cyst with the main pancreatic duct, in our case. Without accurate gross examination of the duct connection, our case could be easily diagnosed as a MCN so that a case of $\mathrm{MCN}$ connecting with the duct could be missed. 
In summary, we report the second pathology confirmed case of MCN communicating with the main pancreatic duct. This communication makes it difficult in differentiating MCNs and IPMNs by image studies preoperatively. The ovarian-type stroma is an important criterium to diagnose a MCN. A careful gross examination and bivalvation of the main duct connecting with the cyst is extremely important to help render the correct diagnosis. If more cases are reported in the future, the pancreatic duct-communicated MCN could become a new entity of pancreatic mucinous neoplasm.

\section{References}

1 Lai J, Fan X, Guindi M, Balzer B and Rutgers J: Endoscopic ultrasound guided - fine needle aspiration (EUS-FNA), in comparison with gross and histologic diagnoses of pancreatic lesions. Am J Digest Dis 1: 68-83, 2014.

2 Farrell JJ: Prevalence, Diagnosis and Management of Pancreatic Cystic Neoplasms: Current Status and Future Directions. Gut and liver 9: 571-589, 2015.

3 Tran Cao HS, Kellogg B, Lowy AM and Bouvet M: Cystic neoplasms of the pancreas. Surg Oncol Clin N Am 19: 267-295, 2010.

4 Adsay NV: Cystic neoplasia of the pancreas: pathology and biology. J Gastrointest Surg 12: 401-404, 2008.

5 Masia R, Mino-Kenudson M, Warshaw AL, Pitman MB and Misdraji J: Pancreatic mucinous cystic neoplasm of the main pancreatic duct. Arch Pathol Lab Med 135: 264-267, 2011.
6 Murakami Y, Uemura K, Ohge H, Hayashidani Y, Sudo T and Sueda T: Intraductal papillary-mucinous neoplasms and mucinous cystic neoplasms of the pancreas differentiated by ovarian-type stroma. Surgery 140: 448-453, 2006.

7 Xiao SY: Intraductal papillary mucinous neoplasm of the pancreas: an update. Scientifica 2012: 893632, 2012.

8 Del Chiaro M, Verbeke C, Salvia R, Kloppel G, Werner J, McKay C, Friess H, Manfredi R, Van Cutsem E, Lohr M and Segersvard R: European experts consensus statement on cystic tumours of the pancreas. Dig Liver Dis 45: 703-711, 2013.

9 Tanaka M, Fernandez-del Castillo C, Adsay V, Chari S, Falconi M, Jang JY, Kimura W, Levy P, Pitman MB, Schmidt CM, Shimizu M, Wolfgang CL, Yamaguchi $\mathrm{K}$ and Yamao K: International consensus guidelines 2012 for the management of IPMN and MCN of the pancreas. Pancreatology 12: 183-197, 2012.

10 Bosman FT, Carneiro F, Hruban RH and Theise ND: WHO Classification of Tumours of the Digestive System. Lyon, International Agency for Research on Cancer (IARC), 2010.

11 Morel A, Marteau V, Chambon E, Gayet B and Zins M: Pancreatic mucinous cystadenoma communicating with the main pancreatic duct on MRI. Brit J Radiol 82: e243-245, 2009.

Received September 11, 2017

Revised September 28, 2017

Accepted September 29, 2017 